\title{
Estadios ninfales de Amnestus rugosus (Hemiptera: Cydnidae)
}

\author{
Jezabel Báez-Santacruz ${ }^{1 *}$ \& María Cristina Mayorga-Martínez ${ }^{2}$ \\ 1. Instituto de Ecología, A. C. Carretera Antigua a Coatepec 351, 91070 Xalapa, Veracruz, México; \\ derophthalma@gmail.com \\ 2. Instituto de Biología, Universidad Nacional Autónoma de México. Apartado postal 70153, 04510 México, D. F., \\ México; mayorgac@ib.unam.mx \\ * Correspondence
}

Recibido 17-II-2016. Corregido 17-VII-2016. Aceptado 19-VIII-2016.

\begin{abstract}
Immature stages of Amnestus rugosus (Hemiptera: Cydnidae). In Mexico there are 29 species of Amnestus, of which six species are from Veracruz. The morphological features and illustrations of immature stages of $A$. rugosus are presented. Notes about the biology and behavior were included. The nymphal and adult stages of A. rugosus were found in remnants of riparian vegetation of the mesophilous forest in La Antigua river basin, Veracruz, Mexico. Twelve sites in five localities were selected and the specimens were sampled in rainy (July) and dry (April) seasons of 2012. The samples were processed in Berlese-Tullgren funnel and Winkler. A total of 419 individuals, 320 adults and 99 nymphs of $A$. rugosus were revised. The size and coloration of the body and punctuation patterns the immature stages of A. rugosus, A. ficus, and A. morelensis are the main differences among these species. A. rugosus is a first record from Veracruz. Rev. Biol. Trop. 65 (1): 31-39. Epub 2017 March 01.
\end{abstract}

Key words: riparian forest, Amnestinae, leaf litter, nymphs, burrowing bugs.

La familia Cydnidae Billberg 1820 comúnmente conocidas como chinches excavadoras, son de cuerpo ovoide y convexo con una longitud entre 2-20 mm; su alimentación está basada en raíces, semillas y jugo de frutas de sus plantas hospederas (Mayorga \& Cervantes, 2006). Los cídnidos están ampliamente distribuidos en el mundo, presentes en regiones tropicales y húmedas (Panizzi \& Grazia, 2015). Su distribución en el mundo está representada por 93 géneros y 750 especies en ocho subfamilias (Grazia, Schuh, \& Wheeler, 2008); en México se han registrado 12 géneros y 39 especies (Mayorga, 2002). La subfamilia Amnestinae Hart se reconoce por poseer un tamaño muy pequeño (1.6-4.5 $\mathrm{mm}$ ) y son reconocidos por la comisura claval extendida que rebasa el ápice del escutelo (Schuh \& Slater, 1995). Existen sólo dos géneros: Amnestus Dallas 1851 y
Lattinestus Eger 2008; el primero es reconocido por su tamaño pequeño y por poseer cuatro o cinco espinas en los juga; mientras que, Lattinestus se distingue por la ausencia de ocelos y la reducida membrana hemielitral (Eger, 2008).

Froeschner (1960) revisó el género Amnestus e incluyó 20 especies distribuidas desde el oeste de Canadá, pasando por Estados Unidos de América, México, América Central, Antillas y hasta América del Sur, en Argentina. En México, se han registrado 29 especies de Amnestus; Mayorga (2002) reconoció la distribución de siete especies para el país, incluyendo la descripción de A. ficus Mayorga \& Cervantes 2001; Mayorga y Cervantes (2005) describieron seis nuevas especies, incluyendo a $A$. rugosus y en 2014 describieron 11 nuevas especies para Chiapas y citaron dos nuevos registros A. bergrothi y A. stali para el estado; 
Mayorga (2015) agrega una nueva especie $A$. morelensis para Morelos. Para Veracruz se han registrado seis especies: A. obscurus Mayorga \& Cervantes 2005, A. ficus, A. pusio (Stål) 1860, A. pusillus Uhler 1876, A. sinuosus Mayorga \& Cervantes 2005 y A. uhleri Distant 1880 .

La mayoría de las especies de Cydnidae son excavadoras con hábitos subterráneos durante casi todo su ciclo de vida, dificultando el conocimiento de su biología, ecología y comportamiento. Mientras que, para las especies de las subfamilias Sehirinae y Amnestinae, que habitan sobre la superficie del suelo, se ha obtenido más información, ya que la alimentación de ninfas y adultos se produce en estructuras aéreas como frutos y semillas de las plantas hospederas (Froeschner, 1960; Mayorga $\&$ Cervantes, 2001). Sin embargo, el tamaño diminuto de las especies de Amnestinae, dificulta su estudio y la información aún es escasa (Panizzi \& Grazia, 2015).

Southwood (1949) describió los estadios inmaduros de Sehirus bicolor Linné y el ciclo de vida de $S$. cinctus (Palisot) fue descrito por Sites y McPherson (1982). Vivan, Nardi, Grazia y Bento (2013) describieron los estadios inmaduros de Scaptocoris carvalhoi Becker asociado principalmente a pastos. Para México, sólo se contaba con notas cortas sobre las características de algunas ninfas (Froeschner, 1960), hasta que se describieron los estadios ninfales de A. ficus (Mayorga \& Cervantes, 2001), Pangaeus (Pangaeus) serripes (Westwood) (Mayorga \& Cervantes, 2014) y A. morelensis (Mayorga, 2015); adicionando información de su asociación con varias especies de Ficus: F. cotinifolia Kunt, F. trigonata L.F. y F. insipida Willd., alimentándose de los frutos y semillas.

En el presente trabajo se describen los estadios ninfales de $A$. rugosus, asociados a la hojarasca de remanentes de vegetación ribereña de bosque mesófilo de montaña en el estado de Veracruz. La especie representa un nuevo registro para el área, ya que sólo se encontraba citada para el estado de Chiapas (Mayorga \& Cervantes, 2005). Se incluyen algunas descripciones sobre la biología y el comportamiento de ninfas y adultos.

\section{MATERIALES Y MÉTODOS}

Área de estudio: Los ejemplares de Amnestus rugosus fueron recolectados en hojarasca asociada a ríos de órdenes entre uno y tres (Strahler, 1964), localizados en 12 sitios pertenecientes a cinco municipios de la zona montañosa del centro de Veracruz, en la parte media de la cuenca del río La Antigua: municipio Atzacán: Matlalapa 19²8'01" N - 9704'39" W 1656 msnm; municipio Coatepec: La Cortadura $19^{\circ} 29^{\prime} 39.5^{\prime \prime} \mathrm{N}-97^{\circ} 02^{\prime} 04.5^{\prime}$ " W, 2007 msnm, propiedad Antonio Guillén 19³1'00" $\mathrm{N}$ - 9700'25.4" W, $1635 \mathrm{msnm}$; municipio Ixhuacán de los Reyes: Puente de Dios $19^{\circ} 23^{\prime} 13.1$ " N - 9704'06.1" W, 1526 msnm y Tlalchi $19^{\circ} 23^{\prime} 14.5^{\prime \prime} \mathrm{N}-97^{\circ} 05^{\prime} 07.9^{\prime \prime} \mathrm{W}$, $1643 \mathrm{msnm}$; municipio Tlalnelhuayocan: Piedra Parada, 19³0'58.6" N - 9700'34.4" W, 1622 msnm, Pixquiac 19³2'04.9" N 96 59'52.3" W, 1573 msnm y Trucha Feliz 19॰31'01.8" N - 9659'12.2” W, 1467 msnm; municipio Xico: Buena Vista, 19²4'03.5” N - 9705'24.9” W, 1854 msnm, Micoxtla $19^{\circ}$ 27' 24" N - 9701'48" W, 1678 msnm, Puente Buenavista $19^{\circ} 24^{\prime} 11.3$ ' N - $97^{\circ} 05^{\prime} 26.1$ " W, 1733 msnm y Xico Viejo $19^{\circ} 27^{\prime} 07^{\prime \prime} \mathrm{N}$ 9703'31" W, 1860 msnm.

Muestreo y procesamiento: Los muestreos se realizaron en la época de lluvias (julio) y secas (abril) de 2012. Se tomaron muestras de hojarasca y se procesaron con embudo de Berlese-Tullgren (apertura de $1 \mathrm{~cm}^{2}$ ), expuestas por $48 \mathrm{~h}$ con una lámpara incandescente de $40 \mathrm{w}$ (Tullgren, 1918). Para la extracción con sacos Winkler, en campo se tamizó la hojarasca con un cernidor de $1 \mathrm{~cm}^{2}$ de apertura, obteniendo tres litros de material cernido. El material fue colocado en bolsas de tela y transportado al laboratorio, donde se depositó en sacos miniWinkler por 96 h (Villareal et al., 2004).

\section{Procesamiento y medición de los ejem-} plares: Los hemípteros recolectados fueron conservados en alcohol al $70 \%$, se separaron y determinaron a nivel de especie los ejemplares del género Amnestus, utilizando las claves 
dicotómicas de Mayorga y Cervantes (2014). Se revisaron un total de 419 individuos, 320 adultos y 99 ninfas. Se tomaron medidas en milímetros de 10 individuos de los estadios ninfales y se obtuvo el promedio con una desviación estándar $( \pm)$. Las ilustraciones fueron realizadas con un microscopio estereoscópico Leica ${ }^{\circledR}$ MZ8 adaptado con un tubo de dibujo. Los individuos fueron depositados en la Colección Entomológica del Instituto de Ecología (IEXA) (Reg. SEMARNAT: Ver. IN. 048.0198). La descripción previa del adulto de
A. rugosus, así como las medidas y el dibujo se encuentran en Mayorga y Cervantes (2005).

\section{RESULTADOS}

Descripciones primer estadio (Fig. 1A): Cuerpo ligeramente alargado; cabeza triangular de color pardo claro con el tylus ancho, rebasando a los juga y con cuatro espinas en el ápice de color pardo oscuro; ojos pequeños de color pardo amarillento; ocelos ausentes; rostro amarillo pálido con cuatro segmentos y

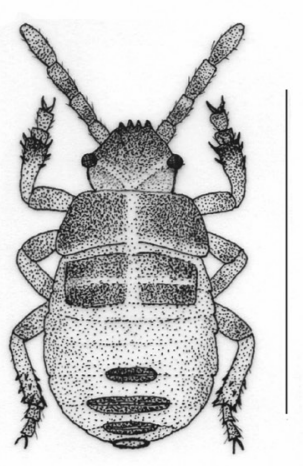

A

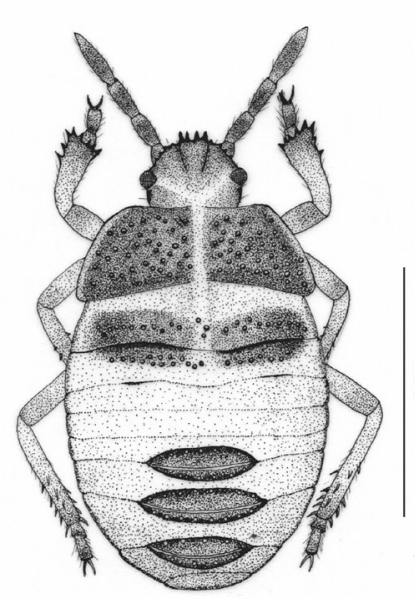

C

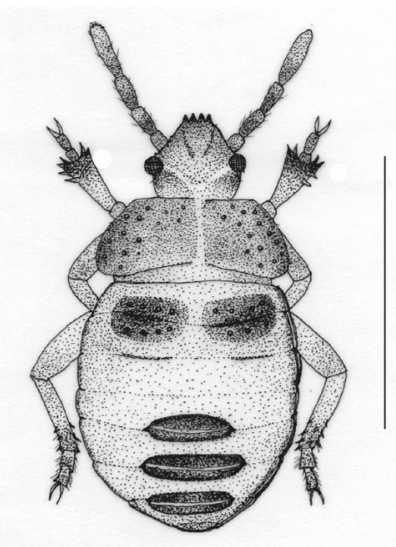

B

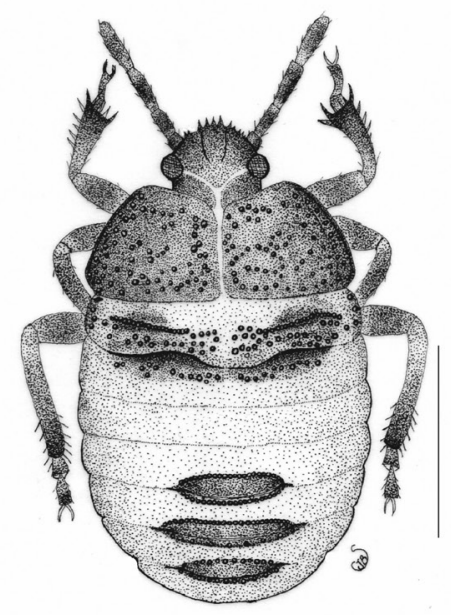

$\mathrm{D}$

Fig. 1. Amnestus rugosus Mayorga \& Cervantes 2005. (A) Primer estadio. (B) Segundo estadio. (C) Tercer estadio. (D) Cuarto estadio $($ Escala $=1 \mathrm{~mm})$.

Fig. 1. Amnestus rugosus Mayorga \& Cervantes 2005. (A) First instar. (B) Second instar. (C) Third instar. (D) Fourth instar $($ Scale $=1 \mathrm{~mm})$. 
sobrepasando las metacoxas; antenas con cuatro artejos el I más pequeño que el II y III de color pardo claro, y el IV más largo y fusiforme amarillo pálido; tórax pardo claro; pronoto más largo que el mesonoto y metanoto; fémures y tibias blanco amarillento, glándulas senescentes, evidentes pardo claro; abdomen, dorsal y ventralmente blanco amarillento.

Medidas $(\mathrm{n}=10)$. Cabeza. Longitud total: $0.23 \pm 0.02$; anchura a través de los ojos: $0.3 \pm$ 0.02 ; distancia interocular: $0.24 \pm 0.02$. Longitud de los artejos antenales: I, 0.08; II, $0.11 \pm$ 0.01 ; III, $0.09 \pm 0.02$; IV, $0.12 \pm 0.01$. Artejos rostrales: I, 0.1; II, 0.1; III, 0.12; IV, 0.12. Pronoto. Longitud total: 0.25 ; anchura a través de los ángulos frontales: 0.42 ; anchura a través de los ángulos humerales: $0.46 \pm 0.4$. Pata posterior: Fémur: 0.15; tibia: 0.2; tarso I: 0.05 ; tarso II: 0.1 . Longitud total del cuerpo: $1.23 \pm 0.17$.

First instar description (Fig. 1A): Slightly elongated body; triangular light browned head with broad tylus, surpassing the juga and with four dark browned spines in the apex; small yellowish-browned pale eyes; ocelli absent; paled yellow four segmented rostrum surpassing the metacoxae; antennae with four segments, number I being smaller than segments II and III, and segment number IV fusiform, all of them with light browned coloration, except IV paled yellow; pale browned thorax; pronotum larger than the mesonotum and metanotum; yellowish paled femora, tibiae, senescent glands and also the abdomen dorsally and ventrally.

Measurements $(\mathrm{n}=10)$. Head. Total length: $0.23 \pm 0.02$; width across eyes: $0.3 \pm 0.02$; interocular distance: $0.24 \pm 0.02$. Antennal segments: I, 0.08; II, $0.11 \pm 0.01$; III, $0.09 \pm 0.02$; IV, $0.12 \pm 0.01$. Rostral segments I, 0.1; II, 0.1 ; III, 0.12; IV, 0.12. Pronotum. Total length: 0.25 ; width across anterior margin: 0.42 ; width across humeral angles: $0.46 \pm 0.4$. Length fore femur: 0.15 ; fore tibia 0.2 ; tarsi I: 0.05 ; tarsi II: 0.1 . Body length: $1.23 \pm 0.17$.

Descripciones segundo estadio (Fig. 1B): Cuerpo ovoide con la anchura máxima a la altura del II segmento abdominal; cabeza jugum y tylus pardo claro, las cuatro espinas en el margen del jugum de color pardo oscuro; ojos pequeños rojizos; tórax pardo obscuro, pronoto con puntuaciones dispersas en su superficie. Mesonoto con una hilera transversal de puntuaciones en la parte media; metanoto con una hilera transversal de puntuaciones en la base de color pardo obscuro; artejos antenales y rostrales amarillo pálido; mesonoto con el margen posterior convexo en la parte media que indica la formación del escutelo; los tres pares de patas color blanco amarillentos a pardo claro; abdomen pardo rosado.

Medidas $(\mathrm{n}=10)$. Cabeza. Longitud total: $0.29 \pm 0.05$; anchura a través de los ojos: 0.34 \pm 0.04 ; distancia interocular: $0.28 \pm 0.04$. Longitud de los artejos antenales: I, 0.1; II, $0.13 \pm$ 0.02 ; III, $0.1 \pm 0.01 ; \mathrm{IV}, 0.16 \pm 0.02$. Artejos rostrales: I, $0.12 \pm 0.01$; II, $0.1 \pm 0.01$; III, 0.18 \pm 0.01 ; IV, $0.15 \pm 0.01$. Pronoto. Longitud total: $0.28 \pm 0.03$; anchura a través de los ángulos frontales: $0.49 \pm 0.03$; anchura a través de los ángulos humerales: $0.74 \pm 0.08$. Pata posterior: Fémur: $0.25 \pm 0.03$; tibia: $0.29 \pm 0.03$; tarso I: 0.05 ; tarso II: $0.12 \pm 0.02$. Longitud total del cuerpo: $1.53 \pm 0.14$.

Second instar description (Fig. 1B): Ovoid body with the maximum width at the second abdominal segment level; light browned head, jugum and tylus; four dark browned spines at the margin of the jugum; reddish small eyes; dark browned thorax; pronotum with disperse punctuations at its surface; mesonotum with a transversal punctuation row at the middle part; metanotum with a transversal dark browned row of punctuations at its base; pale yellowed antennal and rostral segments; convex mesonotum at the posterior margin in the middle part indicating the scutellum formation; yellowish to white legs; pale pinked abdomen.

Measurements $(\mathrm{n}=10)$. Head. Total length: $0.29 \pm 0.05$; width across eyes: $0.34 \pm 0.04$; interocular distance: $0.28 \pm 0.04$. Antennal segments: I, 0.1; II, $0.13 \pm 0.02$; III, $0.1 \pm 0.01$; IV, $0.16 \pm 0.02$. Rostral segments I, $0.12 \pm 0.01$; II, $0.1 \pm 0.01$; III, $0.18 \pm 0.01 ;$ IV, $0.15 \pm 0.01$. 
Pronotum. Total length: $0.28 \pm 0.03$; width across anterior margin: $0.49 \pm 0.03$; width across humeral angles: $0.74 \pm 0.08$. Length fore femur: $0.25 \pm 0.03$; fore tibia $0.29 \pm 0.03$; tarsi I: 0.05 ; tarsi II: 0.12 . Body length: $1.53 \pm 0.14$.

Descripciones tercer estadio (Fig. 1C): Cuerpo piriforme, dorsalmente convexo y con la máxima anchura a la altura del segmento abdominal I; cabeza más ancha que larga de color amarillo pálido, y con el tylus rebasando levemente a los juga, las cuatro espinas en el margen del jugum de color pardo oscuro; rostro alcanzando el ápice de la mesocoxa; pronoto pardo claro con puntuaciones en toda su superficie de color pardo obscuro y con una línea longitudinal que lo cruza amarillo pálido; mesonoto y metanoto con una serie de puntuaciones pardo oscuro hacia la parte media y basal; abdomen amarillo pálido con las tres placas mesiales bien definidas de color pardo obscuro; los tres pares de patas amarillo pálido.

Medidas $(\mathrm{n}=10)$. Cabeza. Longitud total: $0.35 \pm 0.03$; anchura a través de los ojos: 0.38 \pm 0.03 ; distancia interocular: $0.3 \pm 0.02$. Longitud de los artejos antenales: I, $0.07 \pm 0.02$; II, $0.14 \pm 0.02$; III, $0.14 \pm 0.01$; IV, $0.24 \pm$ 0.01 . Artejos rostrales: I, $0.1 \pm 0.02$; II, $0.16 \pm$ 0.02 ; III, $0.2 \pm 0.03$; IV, $0.18 \pm 0.02$. Pronoto. Longitud total: $0.3 \pm 0.03$; anchura a través de los ángulos frontales: $0.5 \pm 0.03$; anchura a través de los ángulos humerales: $0.81 \pm 0.02$. Pata posterior: Fémur: $0.3 \pm 0.02$; tibia: $0.32 \pm$ 0.02 ; tarso I: $0.08 \pm 0.02$; tarso II: $0.14 \pm 0.02$. Longitud total del cuerpo: $1.9 \pm 0.9$.

Third instar description (Fig. 1C): Piriform body, dorsally convex and with the maximum width at the abdominal segment I level; paled yellow head wider than longer, and with the tylus slightly surpassing the juga; four dark browned spines at the margin of the jugum; rostrum reaching the mesocoxae apex; light browned pronotum with dark browned punctuations in all its surface with a series of punctuations in the middle and basal part of the same coloration; pale yellowed abdomen with its three dark browned mesial plates well defined; pale yellowed legs.

Measurements $(\mathrm{n}=10)$. Head. Total length: $0.35 \pm 0.03$; width across eyes: $0.38 \pm$ 0.03 ; interocular distance: $0.3 \pm 0.02$. Antennal segments: I, $0.07 \pm 0.02$; II, $0.14 \pm 0.02$; III, $0.14 \pm 0.01 ; \mathrm{IV}, 0.24 \pm 0.01$. Rostral segments I, $0.1 \pm 0.02$; II, $0.16 \pm 0.02$; III, $0.2 \pm 0.03$; IV $0.18 \pm 0.02$. Pronotum. Total length: $0.3 \pm 0.03$; width across anterior margin: $0.5 \pm 0.03$; width across humeral angles: $0.81 \pm 0.02$. Length fore femur: $0.3 \pm 0.02$; fore tibia $0.32 \pm 0.02$; tarsi I: $0.08 \pm 0.02$; tarsi II: $0.14 \pm 0.02$. Body length: $1.9 \pm 0.9$.

Descripciones cuarto estadio (Fig. 1D): Cuerpo oval, dorsalmente convexo. Cabeza más ancha que larga, de color pardo claro y con el tylus rebasando los juga; las cuatro espinas en el margen del jugum de color pardo oscuro, ojos pardo rojizo; artejos rostrales amarillo pálido, alcanzando la parte basal del metanoto; artejos antenales I-III pardo claro, IV amarillo pálido; pronoto más ancho que largo pardo claro con puntuaciones profundas pardo oscuro en la superficie y con el margen antero lateral liso y carinado y de color pardo claro; mesonoto blanquecino con puntuaciones dispersas en la parte basal; almohadillas alares evidentes; escutelo más ancho que largo cubriendo ligeramente el metanoto; patas anteriores pardo claro; segundo y tercer par pardo amarillento; abdomen blanquecino con las placas mesiales pardo obscuro y levemente rectangulares.

Medidas $(\mathrm{n}=10)$. Cabeza. Longitud total: $0.42 \pm 0.03$; anchura a través de los ojos: 0.48 \pm 0.04 ; distancia interocular: $0.35 \pm 0.03$. Longitud de los artejos antenales: I, $0.11 \pm 0.02$; II, 0.2 ; III, $0.17 \pm 0.03$; IV, $0.24 \pm 0.02$. Artejos rostrales: I, 0.15; II, 0.2; III, $0.23 \pm 0.03$; IV, $0.18 \pm 0.03$. Pronoto. Longitud total: $0.45 \pm$ 0.03 ; anchura a través de los ángulos frontales: $0.62 \pm 0.03$; anchura a través de los ángulos humerales: $1.09 \pm 0.05$. Escutelo. Longitud total: $0.36 \pm 0.04$; anchura máxima: $0.42 \pm$ 0.06 . Pata posterior: Fémur: $0.36 \pm 0.03$; tibia: $0.37 \pm 0.03$; tarso I: 0.15 ; tarso II: 0.2 . Longitud total del cuerpo: $2.41 \pm 0.09$. 
Fourth instar description (Fig. 1D): Oval body, dorsally convex; light browned head wider than longer and with the tylus surpassing the juga; four dark browned spines at the margin of the jugum; pale reddish eyes; pale yellowed rostral segments, reaching the basal part of the metanotum; light browned antennal segments I-III, pale yellowed antennal IV segment; light browned pronotum wider than longer with dark browned deep punctuations at its surface and with the anterolateral light browned margin smooth and carinated; white mesonotum with disperse punctuations at the basal part; wing pads evident; scutellum wider than longer covering slightly the metanotum; light browned anterior legs; paled yellowish middle and third pair of legs; white abdomen with dark browned mesial plates, slightly rectangular.

Measurements $(\mathrm{n}=10)$. Head. Total length: $0.42 \pm 0.03$; width across eyes: $0.48 \pm 0.04$; interocular distance: $0.35 \pm 0.03$. Antennal segments: I, $0.11 \pm 0.02$; II, 0.2 ; III, $0.17 \pm 0.03$; IV, $0.24 \pm 0.02$. Rostral segments I, 0.15; II, 0.2 ; III, $0.23 \pm 0.03 ;$ IV, $0.18 \pm 0.03$. Pronotum. Total length: $0.45 \pm 0.03$; width across anterior margin: $0.62 \pm 0.03$; width across humeral angles: $1.09 \pm 0.05$. Scutellum. Total length: $0.36 \pm 0.04$; width: $0.42 \pm 0.06$. Length fore femur: $0.36 \pm 0.03$; fore tibia $0.32 \pm 0.02$; tarsi I: 0.15; tarsi II: 0.2. Body length: $2.41 \pm 0.09$.

Biología: Amnestus rugosus sólo presenta los primeros cuatro estadios ninfales (I-IV), al igual que la especie A. morelensis (Mayorga, 2015). Las especies del género Amnestus se mantienen en la superficie, ya que en la hojarasca existen lugares óptimos para la oviposición, así como alimento en frutos o semillas disponibles en cada temporada (Mayorga \& Cervantes, 2001). Se registró una mayor abundancia de ninfas en la época seca (241 individuos), siendo el estadio ninfal IV el más abundante, seguido por el estadio III; reflejando una posible preferencia a sustratos secos. Durante las lluvias se registró una menor cantidad de individuos (178), sin embargo, se recolectaron más adultos (141 individuos) que en secas (57 individuos). En ambas épocas, se registraron pocas ninfas de los estadios I-III (Fig. 2).

Distribución: México: Chiapas, Veracruz.

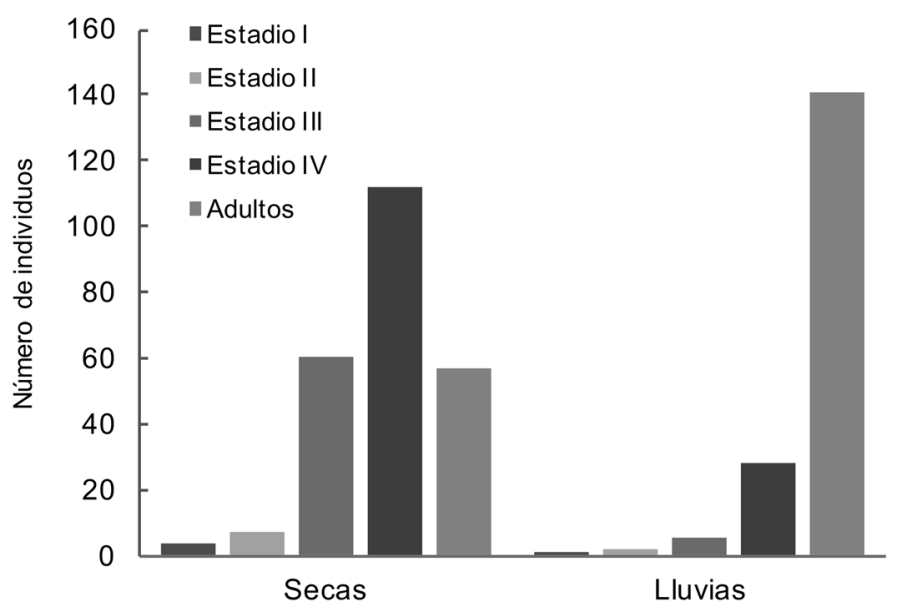

Fig. 2. Abundancia de los estadios ninfales (I, II, III, IV) y adultos de Amnestus rugosus Mayorga \& Cervantes 2005 en los remanentes de vegetación riparia del bosque mesófilo de montaña, Veracruz, México.

Fig. 2. Abundance registered of nymphal stages (I, II, III, IV) and adults of Amnestus rugosus Mayorga \& Cervantes 2005 in remnants of riparian vegetation of the mesophilous forest, Veracruz, Mexico. 


\section{DISCUSIÓN}

La especie Amnestus rugosus representa un nuevo registro para Veracruz, ya que sólo se había reconocido A. ficus (Mayorga \& Cervantes, 2001) para el estado.

Morfológicamente, los estados inmaduros de $A$. rugosus, $A$. ficus y $A$. morelensis son similares; sin embargo, la longitud ( $\mathrm{mm}$ ) del estadio IV para $A$. rugosus es de $2.41 \pm 0.09$, para A. ficus de $2.15 \pm 0.02$, y para A. morelensis de $2.14 \pm 0.08$; además, $A$. rugosus posee puntuaciones en el pronoto, mesonoto y metanoto, mientras que $A$. ficus sólo las tiene en cabeza y pronoto con finas sedas; en $A$. morelensis presenta puntuaciones sólo en el pronoto. Cabeza, pronoto y placas mesiales de $A$. rugosus son de color pardo obscuro y los segmentos abdominales blanquecinos; en $A$. ficus la cabeza, tórax y las placas medias de los segmentos abdominales son rojo-anaranjados; y en A. morelensis, cabeza y pronoto son de color amarillo pálido, y con los segmentos abdominales blanquecinos con las placas medias pardo claro.

Amnestus rugosus sólo presentó cuatro estadios ninfales (I-IV). Estos casos de desarrollo se han reportado, principalmente, en especies acuáticas de la superfamilia Gerroidea (Schuh \& Slater, 1995) y Mesoveloidea (Menke, 1979; Taylor \& McPearson, 1998); recientemente, Mayorga (2015) reportó a $A$. morelensis con este tipo de desarrollo. Las comunidades vegetales ribereñas ofrecen recursos alimentarios abundantes y diversificados para la fauna terrestre (Granados-Sánchez, Hernández, \& López-Ríos, 2006), por ello se podría explicar que $A$. rugosus y A. morelensis, presentan cuatro estadios ninfales, ya que la disponibilidad de alimento es suficiente y no es necesario alargar su ciclo de vida para $\mathrm{su}$ supervivencia. Los bosques ribereños se encuentran entre los ecosistemas más diversos del mundo (Naiman \& Décamps, 1990) y los suelos de estos bosques proveen sitios de forrajeo y de refugio con condiciones ideales para invertebrados que se encuentran en hojarasca, troncos caídos y en detritos de las inundaciones que se acumulan en las zonas ribereñas (Robins \& Cain, 2002).

La mayor abundancia de $A$. rugosus en la época de sequía, se puede explicar debido a que la presencia de lluvias dificulta el movimiento de la especie en el suelo, y además, porque disminuyen los sitios de refugio en especies de hábitos edáficos. La mayor abundancia de individuos tanto de ninfas como adultos, se registró en los meses de abril y mayo, cuando la cantidad de hojas y la producción de semillas en el suelo, son recientes y los sitios de oviposición se vuelven ideales. McColloch y Hayes (1922) mencionan que la distribución, abundancia relativa y actividades estacionales de insectos pueden estar relacionadas con ciertas condiciones del suelo. Observaciones similares fueron registradas por Mayorga y Cervantes (2001) en A. ficus. Así mismo, Tsukamoto y Tojo (1992) mencionan la preferencia de Parastrachia japonensis Scott (Cydnidae) en sitios con mayor hojarasca de árboles huésped, que inicia la caída de las hojas en los meses de abril y mayo; señalando que existe una mayor oviposición y anidación en esa temporada, pues favorece la supervivencia de los individuos.

A pesar de que se encontraron en diferentes tipos de vegetación, las especies de Amnestus registradas para el estado de Veracruz: $A$. ficus y $A$. rugosus se encontraron asociadas a árboles de Ficus con una estacionalidad similar. Amnestus ficus está estrechamente asociada a árboles de Ficus por ello el nombre de la especie, y A. rugosus también ha sido recolectado en estos árboles. Ambas especies están asociadas a la hojarasca por la alta disponibilidad de alimento en frutos y semillas, así como sitios de oviposición y refugio para completar su ciclo de vida.

\section{AGRADECIMIENTOS}

Los autores agradecen a Federico Escobar Sarria por proporcionar los datos de Hemiptera, como parte del proyecto CB 2008-101542-F financiado por CONACYT. Agradecimiento al INECOL para la realización del proyecto biodiversidad y función de ecosistemas ribereños 
en un paisaje fragmentado. Se agradece la colaboración de A. Martínez V., D. Cela C., S. Rocha, N. Portilla, J. Tolome, J. A. Gómez, M. E. Abreo, P. Ronzón y T. Pérez en el trabajo de laboratorio y campo. Agradecemos a Carlos Andrés Rosas Sánchez por la traducción al inglés de las descripciones de los estadios ninfales.

\section{RESUMEN}

En México se han registrado 29 especies del género Amnestus, de las cuales, seis se encuentran en el estado de Veracruz. Se describen e ilustran las características morfológicas de los estadios inmaduros de A. rugosus, incluyendo notas sobre su biología y comportamiento. Las ninfas y adultos fueron recolectados en los remanentes de la vegetación riparia del bosque mesófilo en la cuenca del río La Antigua, Veracruz. El muestreo se realizó en la zona montañosa del centro del estado y en la parte media de la cuenca. Se seleccionaron 12 sitios ubicados en cinco municipios, y se realizaron muestreos en época de lluvias (julio) y en secas (abril); las muestras fueron procesadas en embudos de Berlese-Tullgren y Winkler. Se recolectaron y revisaron 419 ejemplares, 320 adultos y 99 ninfas de $A$. rugosus. El tamaño y coloración del cuerpo y los patrones de puntuación son las principales diferencias entre los estados inmaduros de $A$. rugosus, A. ficus y A. morelensis. $A$. rugosus representa un nuevo registro para Veracruz.

Palabras clave: bosque ribereño, Amnestinae, hojarasca, ninfas, chinches excavadores.

\section{REFERENCIAS}

Billberg, G. J. (1820). Enumeratio insectorum in museo. Stockolm, Sweden: G. J. Billberg.

Eger, J. E. (2008). A new genus and three new species of burrowing bugs (Hemiptera: Heteroptera: Cydnidae: Amnestinae). Proceedings of the Entomological Society of Washington, 110(4), 940-947.

Froeschner, R. C. (1960). Cydnidae of the Western Hemisphere. Proceedings of the United States National Museum, 111(3430), 337-680.

Granados-Sánchez, D., Hernández, M., \& López-Ríos, G. (2006). Ecología de zonas ribereñas. Revista Chapingo, Serie Ciencias Forestales y del Ambiente, 12(1), 55-69.

Grazia, J., Schuh, R. T., \& Wheeler, W. C. (2008). Phylogenetic relationships of family groups in Pentatomoidea based on morphology and DNA sequences (Insecta: Heteroptera). Cladistics, 24, 932-976.
Mayorga, M. M. C., \& Cervantes, P. L. (2001). Life cycle and description of a new species of Amnestus Dallas (Hemiptera-Heteroptera: Cydnidae) associated with the fruit of several species of Ficus (Moraceae) in Mexico. Journal of the New York Entomological Society, 109(3-4), 392-402.

Mayorga, M. M. C. (2002). Revisión genérica de la familia Cydnidae (Hemiptera-Heteroptera) en México, con un listado de las especies conocidas. Anales Instituto de Biología, Universidad Nacional Autónoma de México, Serie Zoológica, 73(2), 157-192.

Mayorga, M. M. C., \& Cervantes, P. L. (2005). Description of six new species of Amnestus Dallas (HemipteraHeteroptera: Cydnidae) from Mexico. Journal of the New York Entomological Society, 113(3-4), 159-173.

Mayorga, M. M. C., \& Cervantes, P. L. (2006). Cydnidae (Hemiptera: Heteroptera) del Centro de Investigaciones Costeras La Mancha, Actopan, Veracruz, México. Revista Mexicana de Biodiversidad, 77, 205-214.

Mayorga, M. M. C., \& Cervantes, P. L. (2014). Immature stages of Pangaeus (Pangaeus) serripes (Westwood) (Hemiptera-Heteroptera: Cydnidae: Cydninae), associated with figs in Mexico. Studies on Neotropical Fauna and Environment, 49(1), 36-40.

Mayorga, M. M. C. (2015). Una especie nueva de Amnestus (Hemiptera: Heteroptera: Cydnidae) de Morelos, México, con descripción de sus estados inmaduros. Revista Mexicana de Biodiversidad, 86, 912-915.

McColloch, J., \& Hayes, W. (1922). The reciprocal relation of soil and insects. Ecological Society of America, 3(4), 288-301.

Menke, A. (1979). The semiaquatic and aquatic Hemiptera of California (Heteroptera: Hemiptera). Bulletin of the California insect survey, 21, 163.

Naiman, R., \& Décamps, H. (1990). The ecology and management of aquatic-terrestrial ecotones. Man and Biosphere Series, 4. Parthernon, UNESCO.

Panizzi, A. R., \& Grazia, J. (2015). True Bugs (Heteroptera) of the Neotropics. Vol. 2. Brazil: Springer.

Robins, J., \& Cain, J. (2002). The past and present condition of the Marsh Creek watershed. Berkley, CA: Natural Heritage Institute.

Schuh, R., \& Slater, J. (1995). True bugs of the World (Hemiptera: Heteroptera), Classification and Natural History. New York, USA: Cornell University Press.

Sites, R. W., \& McPherson, J. (1982). Life history and laboratory rearing of Sehirus cinctus cinctus (Hemiptera: Cydnidae), with description of immature stages. Annals Entomology Society American, 75, 210-215.

Southwood, T. R. (1949). Some notes on the early stages and biology of Sehirus bicolor (L.) (Hemiptera: Cydnidae). The Entomologist's monthly magazine, $85,39-41$. 
Strahler, A. (1964). Quantitative geomorphology of drainage basins and channel networks. En V. Chow (Ed.). Handbook of applied hydrology. New York, USA: Mc Graw-Hill.

Taylor, S., \& McPearson, J. (1998). Laboratory rearing of Mesovelia cryptofila (Heteroptera: Mesoveliidae). Entomological News, 109(2), 95-98.

Tsukamoto, L., \& Tojo, S. (1992). A Report of Progressive Provisioning in a Stink Bug, Parastrachia japonensis (Hemiptera: Cydnidae). Journal of Ethology, 10, 21-29.
Tullgren, A. (1918). Ein sehr einfacher Ausleseapparat fuAr terricole Tierfaunen. Zeitschrift Fur Angewandte Entomology, 4, 149-50.

Villareal, H., Álvarez, M., Córdoba, S., Escobar, F., Fagua, G., Gast, F., ... Umaña, A. (2004). Manual de métodos para el desarrollo de inventarios de biodiversidad. Programa de inventarios de la Biodiversidad. Colombia: Instituto Alexander von Humboldt.

Vivan, L., Nardi, C., Grazia, J., \& Bento, J. (2013). Description of the immatures of Scaptocoris carvalhoi Becker (Hemiptera: Cydnidae). Neotropical Entomology, 42(1), 121-125. 
